\title{
Gamma-rays from Solar Flares
}

\author{
R. Ramaty \\ NASA/GSFC, Greenbelt, MD 20771, U.S.A. \\ N. Mandzhavidze \\ NASA/GSFC and USRA, Greenbelt, MD 20771, U.S.A.
}

\begin{abstract}
Gamma-ray emission is the most direct diagnostic of energetic ions and relativistic electrons in solar flares. Analysis of solar flare gamma-ray data has shown: (i) ion acceleration is a major consequence of flare energy release, as the total flare energy in accelerated particles appears to be equipartitioned between $\gtrsim 1 \mathrm{MeV} /$ nucleon ions and $\gtrsim 20 \mathrm{keV}$ electrons, and amounts to an important fraction of the total energy release; (ii) there are flares for which over $50 \%$ of the energy is in $\alpha$ particles and heavier ions; (iii) in both impulsive and gradual flares, the particles that interact at the Sun and produce gamma rays are essentially always accelerated by the same mechanism that operates in impulsive flares, probably stochastic acceleration through gyroresonant wave particle interaction; and (iv) gamma-ray spectroscopy can provide new information on solar abundances, for example the site of the FIP-bias onset and the photospheric ${ }^{3} \mathrm{He}$ abundance. We propose a new technique for the investigation of mass motion and mixing in the solar atmosphere: the observations of gamma-ray lines from long-term radioactivity produced by flare accelerated particles.
\end{abstract}

\section{Introduction}

Unlike cosmic sources of high-energy emission whose luminosities peak in the hard X-ray and gamma-ray bands (e.g., gamma-ray bursts, Galactic black hole candidates, AGNs), the radiative output of solar flares is maximal in a band ranging from optical to EUV wavelengths (e.g., see Figure 1 in Ramaty \& Mandzhavidze 1993). Nevertheless, there are compelling arguments, based on the overall energetics and impulsiveness of solar-flare hard X-ray and gamma-ray emissions, that flares are fundamentally high-energy phenomena. In the present article, we deal with the gamma-ray emission.

Gamma-ray lines were first observed from solar flares in 1972 with the NaI scintillator on OSO-7 (Chupp et al. 1973), but it was not until 1980 that routine observations of gamma-ray lines and continuum became possible with the much more sensitive NaI spectrometer on the Solar Maximum Mission (SMM; Rieger 1989; Chupp 1990). The SMM detector made important observations during both the declining portion of solar cycle 21 (1980-1984) and the rising portion 
of cycle 22 (1988-1989). Additional gamma-ray line observations during cycle 21 were carried out with a CsI spectrometer on Hinotori (Yoshimori 1990). Recently, a variety of detailed SMM gamma-ray data have become available (Share \& Murphy 1995, 1997, 1998, 1999). During the peak of solar cycle 22, solar-flare gamma-ray observations were carried out with the CGRO instruments OSSE (Murphy et al. 1997), COMPTEL (Ryan \& McConnell 1996), EGRET (Kanbach et al. 1993; Mandzhavidze et al. 1996; Dunphy et al. 1999), and BATSE/CPD (charged particle detector; Ramaty et al. 1994), as well as with the PHEBUS instrument on Granat (Barat et al. 1994) and the gamma-ray spectrometer on Yohkoh (Yoshimori et al. 1994). During the current maximum of cycle 23, both the CGRO and Yohkoh instruments continue to be operational, and, starting in 2000, observations with the imaging Ge spectrometer HESSI (Lin et al. 1998) should become available.

\section{Overview}

Gamma-ray line emission in solar flares results from nuclear de-excitations, neutron capture, and positron annihilation. Narrow de-excitation lines are produced by accelerated protons, $\alpha$ particles, and ${ }^{3} \mathrm{He}$ nuclei interacting with ambient $\mathrm{He}$ and heavier nuclei, while broad lines result from interactions of accelerated $\mathrm{C}$ and heavier nuclei with ambient $\mathrm{H}$ and $\mathrm{He}$. A theoretical nuclear de-excitation spectrum is shown in Figure 1. Starting from high energies, there are strong narrow lines at $6.13 \mathrm{MeV}$ from ${ }^{16} \mathrm{O}, 4.44 \mathrm{MeV}$ from ${ }^{12} \mathrm{C}, 1.79 \mathrm{MeV}$ from ${ }^{28} \mathrm{Si}$, $1.63 \mathrm{MeV}$ from ${ }^{20} \mathrm{Ne}, 1.37 \mathrm{MeV}$ from ${ }^{24} \mathrm{Mg}$, and $0.847 \mathrm{MeV}$ from ${ }^{56} \mathrm{Fe}$. All of these lines result from de-excitations in nuclei of the relatively abundant constituents of the solar atmosphere. This has allowed the determination of the abundances of these elements (Murphy et al. 1991; Ramaty et al. 1995; Ramaty, Mandzhavidze, \& Kozlovsky 1996), showing that, in the gamma-ray production region, the low-FIP (first ionization potential) elements $\mathrm{Mg}, \mathrm{Si}$, and $\mathrm{Fe}$ are enriched relative to $\mathrm{C}$ and $\mathrm{O}$, a result which implies that the FIP bias, known to exist in the corona (e.g., see Reames 1995), already sets in at lower altitudes, presumably in the chromosphere.

Other important lines are formed in reactions between abundant constituents of both the accelerated particles and the ambient medium, leading to excitations in nuclei which are otherwise rare in the solar atmosphere. The feature marked $\alpha \alpha$ is a blend of two lines at 0.429 and $0.478 \mathrm{MeV}$ from ${ }^{7} \mathrm{Be}$ and ${ }^{7} \mathrm{Li}$, respectively. The excited states of these isotopes are populated by interactions of accelerated $\alpha$ particles with ambient He (Kozlovsky \& Ramaty 1974). The line marked ${ }^{3} \mathrm{He}$ at $0.937 \mathrm{MeV}$ and the narrow ${ }^{59} \mathrm{Ni}$ line at $0.339 \mathrm{MeV}$ result, respectively, from interactions of accelerated ${ }^{3} \mathrm{He}$ nuclei with ambient ${ }^{16} \mathrm{O}$ and of accelerated $\alpha$ particles with ${ }^{56} \mathrm{Fe}$, leading to excited states in ${ }^{18} \mathrm{~F}$ and ${ }^{59} \mathrm{Ni}$ (Mandzhavidze, Ramaty, \& Kozlovsky 1997). The line complex marked $\left(\alpha,{ }^{3} \mathrm{He}\right)$ just above $1 \mathrm{MeV}$ consists of 4 lines at 1.04 and $1.08 \mathrm{MeV}$ from the same ${ }^{3} \mathrm{He}$-induced reaction as that which produces the $0.937 \mathrm{MeV}$ line, and at 1.00 and $1.05 \mathrm{MeV}$ due to $\alpha$ particle interactions with ${ }^{56} \mathrm{Fe}$ leading to excited states in ${ }^{58} \mathrm{Co},{ }^{58} \mathrm{Ni}$, and ${ }^{59} \mathrm{Ni}$. This complex was observed, but not resolved, with SMM (Murphy et al. 1990; Share \& Murphy 1998). We anticipate that with the Ge detectors on HESSI such resolution will be possible. 

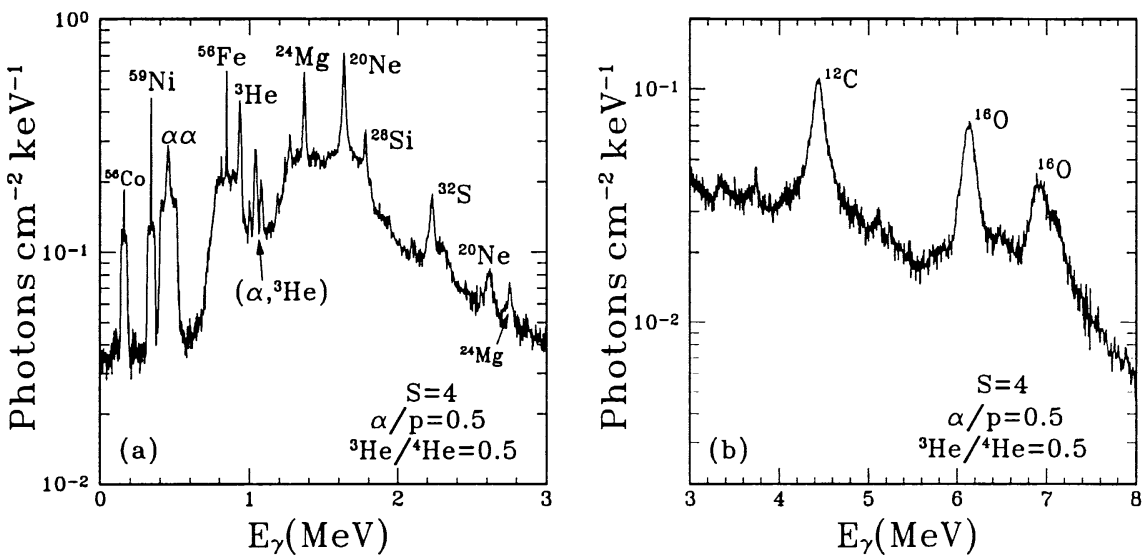

Figure 1. Theoretical nuclear de-excitation gamma-ray spectrum in two energy ranges. The accelerated particles energy spectrum is an unbroken power law with spectral index $S=4$. Impulsive flare abundances are used for the accelerated particles, with $\alpha / \mathrm{p}=0.5$ and ${ }^{3} \mathrm{He} /{ }^{4} \mathrm{He}=0.5$. The ambient medium abundances are those of the average corona, except that $\mathrm{Ne} / \mathrm{O}=0.25$.

These various lines produced by accelerated $\alpha$ particles and ${ }^{3} \mathrm{He}$ nuclei provide information (Mandzhavidze, Ramaty, \& Kozlovsky 1999, hereafter MRK) on the He abundances in both the accelerated particles (the $\alpha / \mathrm{p}$ ratio) and the ambient solar atmosphere, as well as the accelerated particle ${ }^{3} \mathrm{He}$ abundance (the ${ }^{3} \mathrm{He} /{ }^{4} \mathrm{He}$ ratio). We discuss the $\alpha / \mathrm{p}$ ratio in more detail below. Here, we mention that MRK found several flares for which the ambient $\mathrm{He}$ abundance exceeds its photospheric value $(\mathrm{He} / \mathrm{H}=0.084$, Richard et al. 1998), a result that has implications on solar atmospheric dynamics. MRK also showed that several flares require enhanced ${ }^{3} \mathrm{He} /{ }^{4} \mathrm{He}$, meaning that the data definitely require the presence of ${ }^{3} \mathrm{He}$-induced reactions. For some of these flares, ${ }^{3} \mathrm{He} /{ }^{4} \mathrm{He}$ can be as high as 1, while, for essentially all flares for which data are available, values of ${ }^{3} \mathrm{He} /{ }^{4} \mathrm{He}$ as large as 0.1 cannot be ruled out. Such ${ }^{3} \mathrm{He} /{ }^{4} \mathrm{He}$ enhancements are one of the main characteristics of the acceleration mechanism responsible for impulsive, solar energetic particle events (e.g., see Reames 1995). One of the flares (1989 October 24), for which the evidence for enhanced ${ }^{3} \mathrm{He} /{ }^{4} \mathrm{He}$ is particularly strong, belongs to the class of gradual flares, based on the duration of the accompanying soft X-ray emission, showing that, independent of the flare type, in both impulsive and gradual flares, the particles that interact and produce gamma rays are always accelerated by the same mechanism that operates in impulsive flares, namely stochastic acceleration through gyroresonant wave-particle interaction (e.g., see Miller 1998).

The broad lines merge into the underlying emission upon which the narrow lines are superposed. By fitting theoretical gamma-ray spectra to the total observed de-excitation spectra, it was shown (Murphy et al. 1991; Ramaty et al. 1997) that the accelerated heavy ions $\mathrm{Ne}, \mathrm{Mg}, \mathrm{Si}$, and $\mathrm{Fe}$ are enriched relative to $\mathrm{C}$ and $\mathrm{O}-$ an additional characteristic of impulsive flare acceleration. Very 


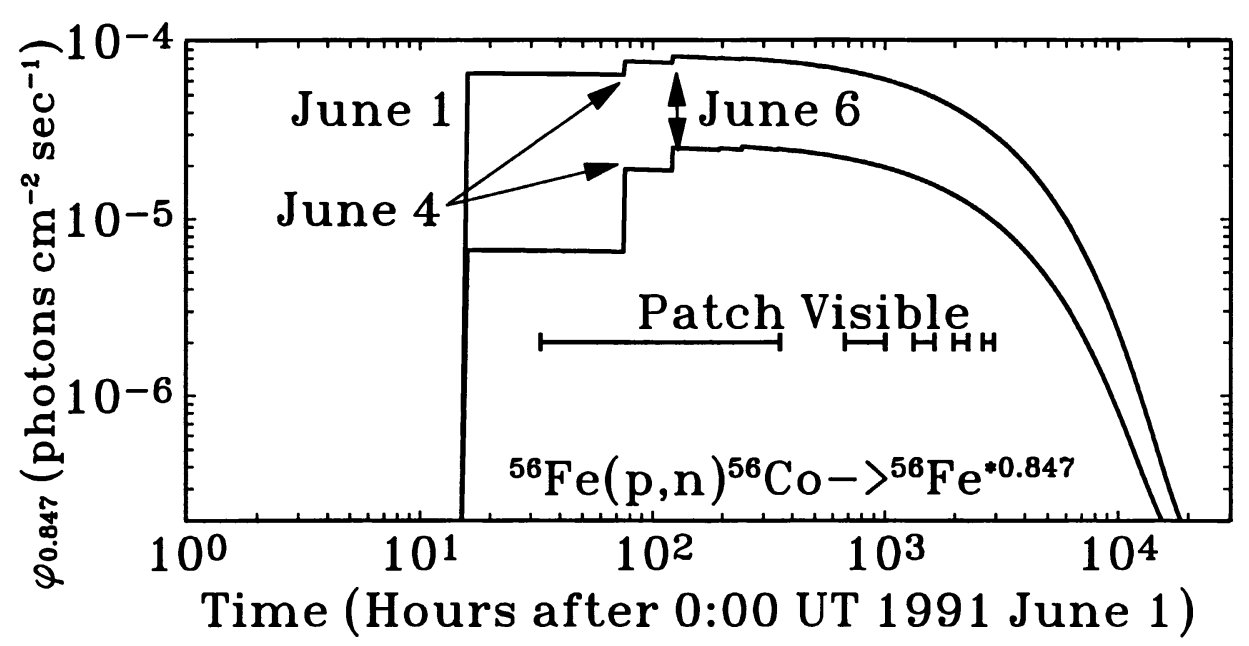

Figure 2. Calculated long term $0.847 \mathrm{MeV}$ line flux resulting from ${ }^{56}$ Co decay produced in the 1991 June flares. The curves correspond to two assumptions for the prompt 4.1-7.6 MeV line fluence which was only partially observed from the June 1 behind-the-limb flare (5600 and 560 photons $/ \mathrm{cm}^{2}$; Ramaty et al. 1997).

recently, individual broad lines have been resolved in a flare-averaged gammaray spectrum (Share \& Murphy 1999), and these also show heavy ion abundance enrichments, in particular for Fe.

The accelerated ions which produce the de-excitation lines also produce neutrons and positrons. Along with the de-excitation lines, the neutrons are produced at sites most likely located in the chromospheric portions of magnetic loops. Some of the neutrons which propagate downward into the photosphere are captured by protons in the photosphere to produce deuterium and essentially monoenergetic photons at $2.223 \mathrm{MeV}$. Thus, because the $2.223 \mathrm{MeV}$ line is formed at a much larger depth than that at which the nuclear reactions take place, the ratio of the $2.223 \mathrm{MeV}$ line fluence to the fluence in the de-excitation lines depends on the position of the flare on the solar disk (Wang \& Ramaty 1974; Hua \& Lingenfelter 1987). The ratio becomes quite small for flares at or near the limb, and can vanish for flares behind the limb. This was demonstrated most dramatically by gamma-ray observations of the 1991 June 1 flare located at $10^{\circ}$ degrees behind the limb for which the $2.223 \mathrm{MeV}$ line was absent while the de-excitation lines were still seen (Barat et al. 1994). Evidently, a considerable fraction of the nuclear reactions occurred in the corona at a site which was visible even though the location of the optical flare was occulted. While this "classical" behavior is reassuring, there is another observation of a flare about $10 \pm 5$ degrees behind the limb for which only the $2.223 \mathrm{MeV}$ line was seen (Vestrand \& Forrest 1993). This flare, on September 29, 1989, was one of the largest on record. Because of the very strong expected attenuation, the observed $2.223 \mathrm{MeV}$ line must have been produced by charged particles interacting on the visible hemisphere of the Sun. It was suggested (Cliver, Kahler, \& 
Vestrand 1993) that these particles were accelerated by a coronal shock over a large volume, thereby producing an extended gamma-ray emitting region visible from the Earth even if the optical flare was behind the limb. Future gamma-ray imaging observations should test this possibility.

In addition to captures on protons, about half the neutrons in the photosphere are captured on photospheric ${ }^{3} \mathrm{He}$ nuclei (Wang \& Ramaty 1974). The flux and time profile of the $2.223 \mathrm{MeV}$ line thus depend on the photospheric ${ }^{3} \mathrm{He}$ abundance, allowing the derivation of its abundance (Hua \& Lingenfelter 1987). By combining the results with the photospheric $\mathrm{He} / \mathrm{H}$ obtained from helioseismology (Richard et al. 1998), we provided evidence that the photospheric ${ }^{3} \mathrm{He} /{ }^{4} \mathrm{He}$ could be lower than the corresponding solar wind value, suggesting the possible isotopic fractionation of $\mathrm{He}$ in the process of solar wind acceleration (Mandzhavidze \& Ramaty 1998).

Positron annihilation leads to the line at $0.511 \mathrm{MeV}$. The positrons result from the decay of various radioactive nuclei produced by the accelerated particles (Kozlovsky, Lingenfelter, \& Ramaty 1987), as well as from the decay of muons resulting from pions which are produced by the highest-energy accelerated particles (Murphy, Dermer, \& Ramaty 1987). The $0.511 \mathrm{MeV}$ line is accompanied by a continuum extending up to $0.511 \mathrm{MeV}$, resulting from positronium annihilation. The ratio of the flux in this continuum to the flux in the $0.511 \mathrm{MeV}$ line, as well as the width of the line, depends on the density, temperature, and state of ionization of the ambient gas at the annihilation site (e.g., see Guessoum, Ramaty, \& Lingenfelter 1991). Future observations of positron annihilation radiation with imaging spectrometers of high spectral resolution (e.g., HESSI) should thus provide interesting information on the properties on the flaring solar atmosphere.

The line emission is superposed on a continuum dominated by bremsstrahlung produced by primary electrons accelerated out of the ambient atmosphere. The ratio of the fluence in the lines to that in the bremsstrahlung was used to determine the electron-to-proton ratio $(e / p)$ (Ramaty et al. 1993). The derived values were compared with the values of $e / p$ obtained from observations of solar flare particle events in interplanetary space. The gamma-ray results reveal comparable or even higher values of $e / p$ than the impulsive events in space, regardless of whether the flare is impulsive or gradual. This is consistent with the ${ }^{3} \mathrm{He}$ result for the one flare mentioned above and the heavy element abundance enhancements found in two other gradual flares, 1981 April 27 (Murphy et al. 1981) and June 1, 1991 (Ramaty et al. 1997).

In the energy range above $10 \mathrm{MeV}$, along with the bremsstrahlung from primary electrons, there can also be a significant contribution from pion decay radiation which was observed up to energies exceeding about $1 \mathrm{GeV}$ during the 1991 June 11 and June 15 flares (Akimov et al. 1991; Kanbach et al. 1993). The striking feature of these $\mathrm{GeV}$ gamma-ray observations is their long duration, particularly for the June 11 flare for which the gamma-ray emission lasted for about 8 hours. It is still not known whether the particles that produced the pions were accelerated in the impulsive phase of the flare and subsequently trapped in magnetic loops at the Sun or continuously accelerated over the duration of the emission (see Mandzhavidze et al. 1996). 

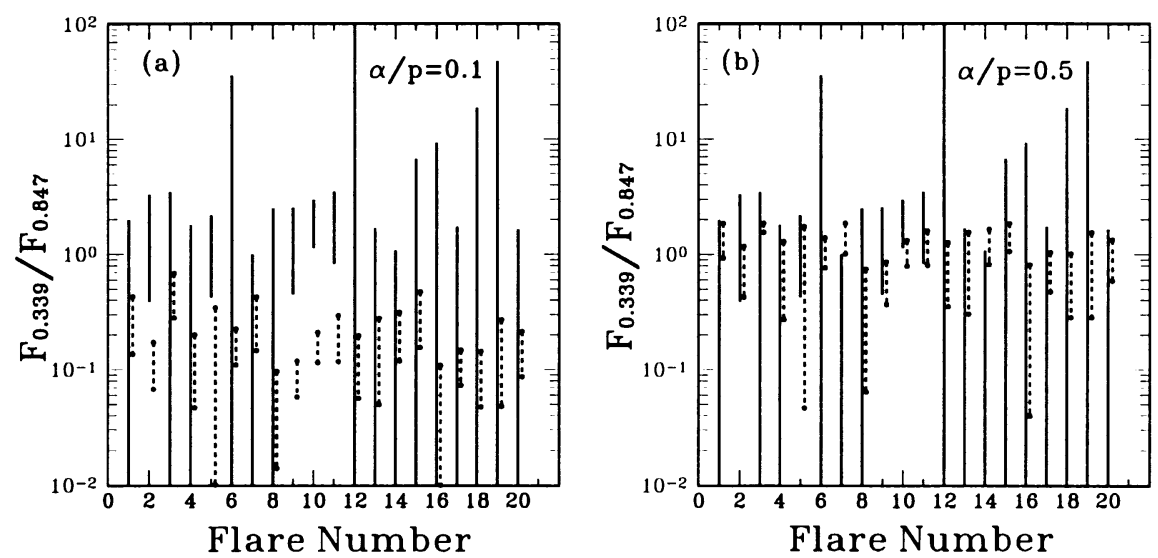

Figure 3. Observed (solid bars) and calculated (dashed bars) fluence ratios of the $0.339 \mathrm{MeV}$ and $0.847 \mathrm{MeV}$ lines. Both these lines result from interactions in $\mathrm{Fe}$ targets. The calculated ratios depend on $\alpha / \mathrm{p}$, panels (a) and (b), showing that $\alpha / \mathrm{p}>0.1$ for flares $2,5,9,10$, and 11 .

\section{Long-Duration Gamma-Ray Line Emission from Radionuclei}

Very long duration gamma-ray line emission is expected from de-excitations of nuclei resulting from the decay of long-lived radionuclei that are themselves produced by the accelerated particles. The most promising such line is at $0.847 \mathrm{MeV}$ resulting from ${ }^{56} \mathrm{Co}$ decay into ${ }^{56} \mathrm{Fe}$ (half-life 78.8 days). The ${ }^{56} \mathrm{Co}$ is produced in $(p, n)$ reactions of both accelerated protons with ambient $\mathrm{Fe}$ and accelerated $\mathrm{Fe}$ with ambient $\mathrm{H}$. Figure 2 shows the time dependence of the emission that would have been expected after the series of large flares of June 1991. The two curves correspond to two different values for the total prompt de-excitation line emission of the 1991 June 1 flare, which is uncertain as only the coronal part of the gamma-ray source was observed, as mentioned above. The $0.847 \mathrm{MeV}$ line is accompanied by additional lines, also resulting from ${ }^{56} \mathrm{Co}$ decay, at 1.238 and $2.599 \mathrm{MeV}$ with fluxes of $68 \%$ and $17 \%$ relative to the $0.847 \mathrm{MeV}$ line flux plotted in the figure. Even though both proton and Fe interactions produce these ${ }^{56}$ Co decay lines, the lines are very narrow because the ${ }^{56} \mathrm{Co}$ nuclei are stopped by energy losses in the solar atmosphere before they decay. Such long-duration, very narrow lines from flare-produced radioactivity have not yet been observed. Their detection, hopefully with HESSI which could measure the size and development of the radioactive patch, would provide unique information on mixing and transport processes in the solar atmosphere.

\section{Accelerated $\alpha$ Particle Abundances}

A detailed flare-by-flare derivation of $\alpha / \mathrm{p}$ was provided in MRK. The key linefluence ratio is $F_{0.339} / F_{0.847}$, plotted for 20 flares in Figure 3 . Since both the narrow 0.339 and $0.847 \mathrm{MeV}$ lines result from the same ${ }^{56} \mathrm{Fe}$ target, this line ratio is independent of the ambient medium composition. However, it does depend on 

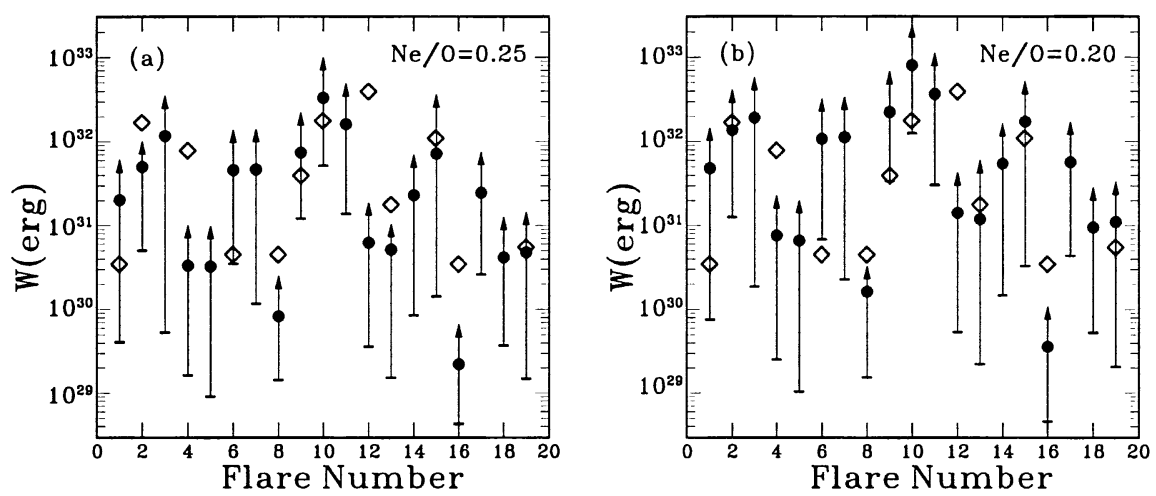

Figure 4. Calculated energy contents in ions and electrons. The solid circles and the lower limits indicated by horizontal bars are, respectively, the ion energy contents assuming low energy cutoffs at $1 \mathrm{MeV} /$ nucleon and at the maximum $E_{c}$ allowed by the observed Ne-to$\mathrm{O}$ line-fluence ratios. Open diamonds are the electron energy content, assuming a cutoff at $20 \mathrm{keV}$.

$\alpha / \mathrm{p}$, because only $\alpha$ particles produce the $0.339 \mathrm{MeV}$ line, while both protons and $\alpha$ 's produce the line at $0.847 \mathrm{MeV} . F_{0.339} / F_{0.847}$ also depends on the spectrum of the accelerated particles which is determined from the $1.63 \mathrm{MeV}\left({ }^{20} \mathrm{Ne}\right)$ to-6.13 MeV $\left({ }^{16} \mathrm{O}\right)$ line-fluence ratio (see $\mathrm{MRK}$ for more details). It can be seen from Figure $3 \mathrm{a}$ that if $\alpha / \mathrm{p}=0.1$ the measured ratios (solid bars) exceed the calculated values (dashed bars) for 5 flares (numbers 2, 5, 9, 10,and 11), but for $\alpha / p=0.5$, the calculated and observed ratios for these flares become consistent, showing that for these 5 flares $\alpha / \mathrm{p}$ exceeds its canonical value of 0.1 . The large errors in the measured $F_{0.339} / F_{0.847}$ for the other flares prevents the derivation of $\alpha / \mathrm{p}$ for these flares. The possibility of determining the accelerated $\alpha / \mathrm{p}$ and its variation with time during the acceleration process from future, more detailed gamma-ray data will be an important ingredient for the understanding of solar-flare particle acceleration (Miller 1998).

\section{Energy Content in the Gamma-Ray Producing Ions}

To account for the strong $1.63 \mathrm{MeV}{ }^{20} \mathrm{Ne}$ line in terms of an acceptable $\mathrm{Ne}$ to-O abundance ratio, the accelerated proton and $\alpha$ particle energy spectra must be extended down to almost $1 \mathrm{MeV} /$ nucleon to take advantage of the lowenergy threshold for the production of the $1.63 \mathrm{MeV}$ line. This extension has modified the previous paradigm that the energy content in ions, $W_{i}$, is smaller than $W_{e}$, the energy contained in the hard X-ray producing electrons whose energy spectrum is thought to extend down to about $20 \mathrm{keV}$ where it merges with the high-energy tail of the thermal electron distribution. That $W_{i} \ll$ $W_{e}$ followed from the assumption that the ion energy spectrum is given by the Bessel function appropriate for steady-state stochastic acceleration (Ramaty 1986). The fact that this spectrum is quite hard at energies less than about 
$10 \mathrm{MeV} /$ nucleon led to a relatively small value for $W_{i}$, but it also required that $\mathrm{Ne} / \mathrm{O} \gtrsim 0.3$ (Murphy et al. 1991). This conflicted with the coronal value of $\simeq 0.15$, which is also the generally accepted photospheric value. However, the gamma-ray result was considered acceptable because it pertained to only one flare. When similar data became available for many flares (Share \& Murphy 1995), the assumption of the Bessel function spectrum was abandoned in favor of an unbroken power law down to $1 \mathrm{MeV} /$ nucleon, leading to much larger ion energy contents (Ramaty et al. 1995). Nevertheless, if $\mathrm{Ne} / \mathrm{O}$ in the gamma-ray production region were $<0.2$, the implied ion spectrum would be too steep to account for the observed $2.223 \mathrm{MeV}$ neutron capture line fluxes (see Ramaty et al. 1996). In MRK, therefore, calculations were done for two values of $\mathrm{Ne} / \mathrm{O}$, 0.20 and 0.25 , where the lower value implied steeper ion spectra.

To investigate this issue further, we assumed here that the accelerated ion spectrum is a power law down to a break energy $E_{c}$, below which it is flat down to zero energy. We used the previously derived power-law indices for $\mathrm{Ne} / \mathrm{O}=0.25$ and 0.2 , and calculated the maximal value of $E_{c}$ that is still consistent with the measured $F_{1.63} / F_{6.13}$. This maximal $E_{c}$ can be as low as $2.5 \mathrm{MeV} /$ nucleon for some flares. We then calculated $W_{i}$ taking $\alpha / \mathrm{p}=0.1$ for all the flares, except for flares $2,5,9,10$, and 11 for which $\alpha / \mathrm{p}=0.5(\S 4)$. We also included the energy contained in accelerated $\mathrm{C}$ and heavier nuclei, assuming an accelerated $\mathrm{He} / \mathrm{O}$ of 46 (Reames 1995) and taking typical impulsive-flare heavy-ion enrichments relative to $\mathrm{O}$. We note that, when $\alpha / \mathrm{p}=0.5$, over $75 \%$ of the ion energy content is in $\alpha$ particles and heavier nuclei.

The results are shown in Figure 4 for two values of $\mathrm{Ne} / \mathrm{O}$ (which determine the spectral indices). The solid circles are for $E_{c}=1 \mathrm{MeV} /$ nucleon, while the horizontal bars, which denote the lower limits on $W_{i}$, are for maximal $E_{c}$. Also shown is the energy content $W_{e}$ in the $>20 \mathrm{keV}$ electrons that we calculate (open diamonds) based on hard X-ray data (R. Schwartz 1996, private comm.). We first note that because the ion spectra are steeper for the smaller $\mathrm{Ne} / \mathrm{O}$, $W_{i}$ for $\mathrm{Ne} / \mathrm{O}=0.2$ is larger than for $\mathrm{Ne} / \mathrm{O}=0.25$. The comparison of $W_{i}$ for $E_{c}=1 \mathrm{MeV} /$ nucleon with $W_{e}$ reveals an approximate equipartition between the energy contents in $\gtrsim 20 \mathrm{keV}$ electrons and $\gtrsim 1 \mathrm{MeV} /$ nucleon ions. The introduction of a break in the ion energy spectrum above $1 \mathrm{MeV} /$ nucleon obviously lowers the derived values of $W_{i}$. The upward pointing arrows suggest the possibility of even larger $W_{i}$ if the ion energy spectra would continue as unbroken power laws to energies below $1 \mathrm{MeV} /$ nucleon.

\section{References}

Akimov, V. V., et al. 1991, in Proc. of 22nd ICRC, eds. D. O'Sullivan et al. (Dublin: Dublin Institute for Advanced Studies), 3, 73

Barat, C., et al. 1994, ApJ, 425, L109

Chupp, E. L. 1990, Physica Scripta, T18, 15

Chupp, E. L., et al. 1973, Nature, 241, 333

Cliver, E. W., Kahler, S. W., \& Vestrand, W. T. 1993, Proc. of 23rd ICRC, eds. D. A. Leahy (Calgary: University of Calgary), 3, 91

Dunphy, P. P., et al. 1999, Solar Phys., 187, 45 
Guessoum, N., Ramaty, R., \& Lingenfelter 1991, ApJ, 378, 170

Hua, X.-M., \& Lingenfelter, R. E. 1987, Solar Phys., 107, 351

Kanbach, G., et al. 1993, A\&AS, 97, 349

Kozlovsky, B., Lingenfelter, R. E., \& Ramaty, R. 1987, ApJ, 316, 801

Kozlovsky, B., \& Ramaty, R. 1974, ApJ, 191, L43

Lin, R. P., et al. 1998, in Proc. of SPIE, 3442, Missions to the Sun II, ed. C. M. Korendyke (Bellingham: SPIE), 2

Mandzhavidze, N., \& Ramaty, R. 1998, in Highlights of Astronomy, ed. J. Andersen (Dordrecht: Kluwer), 11B, 759

Mandzhavidze, N., Ramaty, R., Bertsch, D. L., \& Schneid, E. J. 1996, in AIP Conf. Proc., 374, High Energy Solar Physics, eds. R. Ramaty, et al. (New York: AIP), 225

Mandzhavidze, N., Ramaty, R., \& Kozlovsky, B. 1997, ApJ, 489, L99

Mandzhavidze, N., Ramaty, R., \& Kozlovsky, B. 1999, ApJ, 518, 918

Miller, J. A. 1998, Space Sci. Rev., 86, 79

Murphy, R. J., Dermer, C. D., \& Ramaty, R. 1987, ApJS, 63, 721

Murphy, R. J., et al. 1997, ApJ, 490, 883

Murphy, R. J., Ramaty, R., Kozlovsky, B., \& Reames, D. V. 1991, ApJ, 371, 793

Murphy, R. J., Share, G. W., Letaw, J. R., \& Forrest, D. J. 1990, ApJ, 358, 298

Ramaty, R. 1986, in The Physics of the Sun, ed. P. A. Sturrock (Dordrecht: Reidel), 293

Ramaty, R., \& Mandzhavidze, N. 1993, in AIP Conf. Proc., 280, Compton Gamma Ray Observatory, eds. M. Friedlander et al. (New York: AIP), 643

Ramaty, R., Mandzhavidze, N., Barat, C., \& Trottet, G. 1997, ApJ, 479, 458

Ramaty, R., Mandzhavidze, N., \& Kozlovsky, B. 1996, in AIP Conf. Proc., 374, High Energy Solar Physics, eds. R. Ramaty et al. (New York: AIP), 172

Ramaty, R., Mandzhavidze, N., Kozlovsky, B., \& Murphy, R. J. 1995, ApJ, 445, $\mathrm{L} 193$

Ramaty, R., Mandzhavidze, N., Kozlovsky, B., \& Skibo, J. G. 1993, Adv. Space Res., 13, no. 9, 275

Ramaty, R., Schwartz, R. A., Enome, S., \& Nakajima, H. 1994, ApJ, 436, 941

Reames, D. V. 1995, Adv. Space Res., 15, no. 7, 41

Richard, O., et al. 1998, A\&A, 338, 756

Rieger, E. 1989, Solar Phys., 121, 323

Ryan, J. M., \& McConnell, M. M. 1996, in AIP Conf. Proc., 374, High Energy Solar Physics, eds. R. Ramaty et al. (New York: AIP), 200

Share, G. H., \& Murphy, R. J. 1995, ApJ, 452, 933

Share, G. H., \& Murphy, R. J. 1997, ApJ, 485, 409

Share, G. H., \& Murphy, R. J. 1998, ApJ, 508, 876

Share, G. H., \& Murphy, R. J. 1999, in Proc. of 26th ICRC, eds. D. Kieda, M. Salamon, \& B. Dingus (Salt Lake City: University of Utah), 6, 13 
Vestrand, W. T., \& Forrest, D. J. 1993, ApJ, 409, L69

Wang, H. T., \& Ramaty, R. 1974, Solar Phys., 36, 129

Yoshimori, M. 1990, ApJS, 73, 227

Yoshimori, M. 1994, ApJS, 90, 639 